https://doi.org/10.15407/ujpe64.7.640

M. SCHMIDT

on behalf of the PANDA collaboration

II. Physikalisches Institut, Justus Liebig University Giessen

(16, Heinrich-Buff-Ring, 35392 Giessen, Germany; e-mail:

mustafa.a.schmidt@exp2.physik.uni-giessen.de)

\title{
THE PANDA DETECTOR AT FAIR
}

PANDA is a fixed-target experiment that is going to address a wide range of open questions in the hadron physics sector by studying the interactions between antiprotons with high momenta and a stationary proton target. The PANDA detector is currently under construction and will be situated in the HESR that is a part of the future FAIR accelerator complex on the area of the GSI Helmholtzzentrum für Schwerionenforschung in Darmstadt. The key features of the detector are: the precise tracking in strong magnetic fields, excellent particle identification, and high-resolution calorimeters.

Ke ywords: FAIR, PANDA, antiprotons.

\section{Introduction}

\subsection{Antiproton Production at FAIR}

The future Facility for Antiproton and Ion Research (FAIR) is designed as an extension to the existing GSI Helmholtzzentrum für Schwerionenforschung in Darmstadt, Germany. A new linear accelerator (p-LINAC) that is currently under development will accelerate protons up to a kinetic energy of $70 \mathrm{MeV}$. After being further accelerated in the two synchrotrons SIS18 and SIS100, these protons will be extracted to collide with a nickel target. The antiprotons, that are created during this process, will be collected by the Collection Ring (CR) and further injected into the High Energy Storage Ring (HESR) where the PANDA detector will be located. In addition to NUSTAR, CMB, and APPA, it is going be one of the four excellent physical experiments at FAIR [2].

\subsection{HESR \& PANDA}

The injection momentum of the antiprotons into the HESR will be $3.8 \mathrm{GeV} / \mathrm{c}$. Inside the HESR, the beam momentum can be modified to values between $1.5 \mathrm{GeV} / \mathrm{c}$ and $15 \mathrm{GeV} / \mathrm{c}$. One of the key features of the HESR is the stochastic cooling that can be applied over the full momentum range. In addition to

(C) M. SCHMIDT, 2019 that, the HESR can run in two different modes: a high-luminosity and a high-resolution mode. The important parameters of both modes are represented in the table below. The high-luminosity mode with an interaction rate of $20 \mathrm{MHz}$ will not be available in the beginning, because it requires an additional synchrotron called Recycling Energy Storage Ring (RESR). The investigation of collisions between antiprotons and protons in PANDA will be used to answer open questions in the fields of nucleon structure, hadron spectroscopy, and nuclear physics.

Because of the forward boost of the created particles, PANDA will consist of two spectrometers: a target spectrometer designed as an onion shell detector around the interaction point and a forward spectrometer covering small polar angles. Both spectrometers have redundant detector systems for the tracking, particle identification (PID), and calorimetry. The complete PANDA detector including all sub-

Different operation modes of the HESR

\begin{tabular}{|l|c|c|}
\hline \multicolumn{1}{|c|}{ Parameter } & High Res. & High Lum. \\
\hline Momentum $[\mathrm{GeV} / \mathrm{c}]$ & $1.5-15$ & $1.5-15$ \\
Antiprotons & $10^{10}$ & $10^{11}$ \\
Luminosity $\left[\mathrm{cm}^{2} \mathrm{~s}^{-1}\right]$ & $2 \times 10^{31}$ & $2 \times 10^{32}$ \\
Resolution $\Delta p / p$ & $5 \times 10^{-5}$ & $1 \times 10^{-4}$ \\
\hline
\end{tabular}

ISSN 2071-0194. Ukr. J. Phys. 2019. Vol. 64, No. 7 


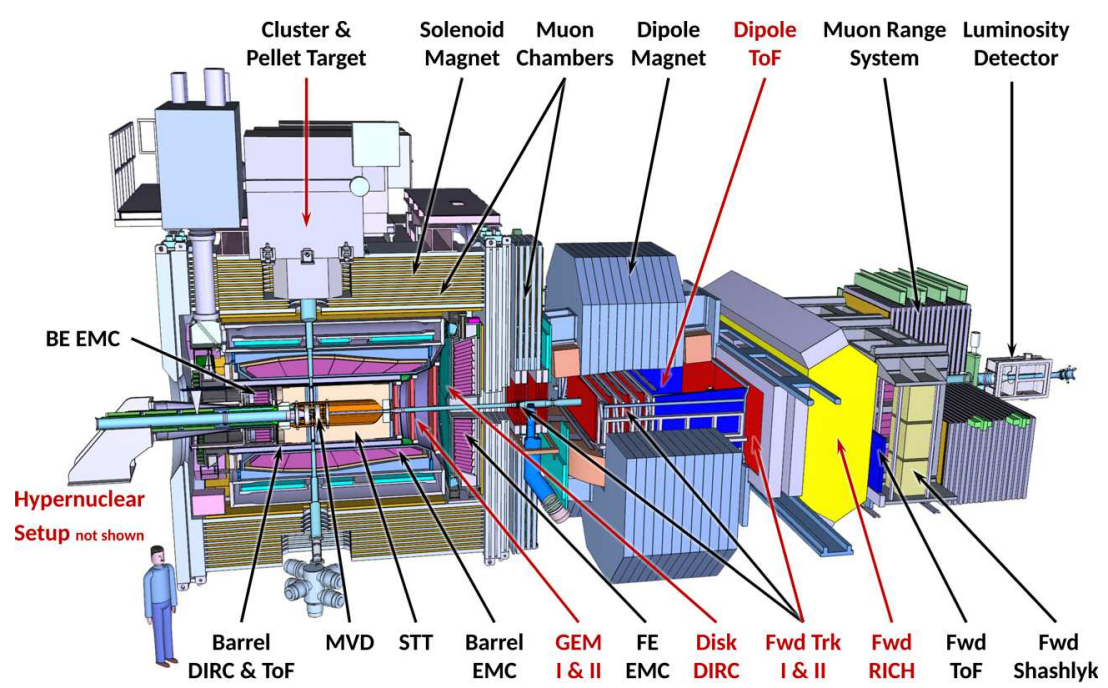

Fig. 1. The PANDA detector at FAIR including all detectors for phase 1 (black) and phase 2 (gray)

detectors is shown in Fig. 1. For most of the physical analysis, it is important to cover the full solid angle with all subdetectors.

\subsection{Physics programs}

The PANDA experiment is designed to cover a large amount of antiproton physics programs in order to answer many open questions related to the huge sector of hadron physics in the non-perturbative region [7]. This can be summarized as follows:

- Hadron spectroscopy: Production of exotic QCD states and exploring charm hadrons.

- Nucleon structure: Investigating the generalized parton structure and time-like form factors.

- Nuclear physics: Studying hadrons in nuclei and performing hypernuclear physics.

\subsection{Time schedule}

The time schedule of PANDA is divided into 3 phases. In the present phase 0 , the subdetectors of PANDA are under development and tested in various other excellent High Energy Physics (HEP) experiments. With the availability of the PANDA hall in 2022 , the installation of phase 1 subdetectors is going to start. In the year 2025, a proton beam will be used for commissioning the start setup of PANDA, whereas the first antiproton beam will be available in 2026. The measurements of phase 2 that includes all remaining subdetectors are going to be started

ISSN 2071-0194. Ukr. J. Phys. 2019. Vol. 64, No. 7

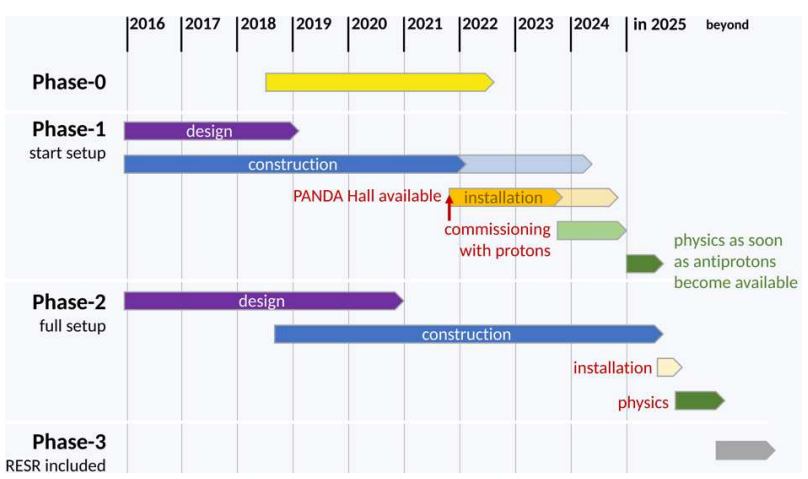

Fig. 2. The different phases of the PANDA detector

around 2027. After running for a long-time period, further physical experiments with other possible setups can be performed, e.g., phase 3 that represents the high-luminosity mode. A complete overview over all phases is presented in Figure 2.

\section{Proton Target}

The proton target marks the interaction point (IP) and can be seen as the core component of a PANDA target spectrometer. Two targets are currently foreseen. The clusterjet target will be already installed in the first phase of PANDA. It creates small hydrogen clusters with a size of $10^{3}$ to $10^{5}$ atoms per cluster by expanding pre-cooled and compressed hydrogen into the vacuum of the beam pipe. 
The pellet target creates small droplets of frozen hydrogen with pellet diameters between 10 and $30 \mu \mathrm{m}$ in a triple point chamber. These pellets will be injected into the target tube with a falling speed of around $60 \mathrm{~m} / \mathrm{s}$. In total, a constant flow rate of 100,000 pellets per second can be achieved. Further targets are under development and can be installed in later phases.

\section{Magnets}

For the purpose of the momentum determination, two magnets will be installed in PANDA [3]. A solenoid magnet in a target spectrometer will create a field of around $2 \mathrm{~T}$ with a field inhomogenity of less than $2 \%$ over the full field map. Inside the iron yoke, drift tube chambers will be placed for PID and tracking of high energetic muons. The inner/outer diameter of the magnet will be $1.9 \mathrm{~m} / 2.3 \mathrm{~m}$. The length is given by $4.9 \mathrm{~m}$, which leads to a total magnet weight of approx. $360 \mathrm{t}$.

In the forward spectrometer, a dipole magnet is going to create a magnetic field of $1 \mathrm{~T}$. The length of the magnet is $2.5 \mathrm{~m}$ and has an overall weight of $240 \mathrm{t}$. It has a conical shape with an opening diameter of $1 \mathrm{~m}$ at the front- and $3 \mathrm{~m}$ at the backside. The key feature of this magnet is a short ramping speed of $1.25 \%$ per second and the possibility for a synchronized operation with the HESR.

\section{Tracking}

\subsection{Micro Vertex Detector}

The inner-most detector and the closest one to the target is the Micro Vertex Detector (MVD) [4]. It consists of four barrels around the interaction point and six disks in the forward direction. The inner barrel layers of the MVD contain hybrid pixels with a size of $100 \mu \mathrm{m} \times 100 \mu \mathrm{m}$, while the outer layers are made of double sided microstrips. The last two disks will be equipped with pixel and strip detectors.

A time resolution of $6 \mathrm{~ns}$ and a pixel resolution of $28 \mu \mathrm{m}$ are achievable. From these values, a vertex resolution of $50 \mu \mathrm{m}$ can be computed. This high vertex resolution is important to measure displaced vertices, e.g., to analyze different decay channels in the open charm sector.

\subsection{Straw tube tracker}

The Straw Tube Tracker (STT) [5] is placed in a cylindrical shape around the MVD and consists of
4,200 Al-Mylar drift tubes filled with a mixture of $\mathrm{Ar} / \mathrm{CO}_{2}$ gas. The readout can be done with ASICs combined with TDCs or with Flash ADCs (FADCs) instead. In total, 21 to 27 layers are planned to be installed, of which 8 layers are skewed by $3^{\circ}$ for the purpose to reconstruct the longitudinal coordinate. The electron avalanche gain of these tubes is about 100. The inner/outer radius of the detector is $15 \mathrm{~cm} / 42 \mathrm{~cm}$. Each tube has a diameter of $1 \mathrm{~cm}$ and a length of $150 \mathrm{~cm}$. Taking the spatial and time resolutions into account, a $\varrho / \phi$ plane resolution of $150 \mu \mathrm{m}$ and a $z$ resolution of $1 \mathrm{~mm}$ can be achieved. Currently, most of the tubes have been produced and already been mounted within an STT prototype.

\subsection{Gas electron multiplier tracker}

The Gas Electron Multiplier (GEM) tracker in the forward region of the target spectrometer is a combination of three stations. Two stations will be installed in phase 1 and the third one in phase 2. The foreseen large area GEMs were developed at CERN and are going to be produced in Poland. They contain a $50 \mu \mathrm{m}$ kapton layer covered by thin copper layers with a thickness of 2 to $5 \mu \mathrm{m}$ on both sides. The ADCs, that are planned for the readout, will allow for the cluster centroiding to calculate the precise particle position and to reach a position resolution of better than $100 \mu \mathrm{m}$.

\subsection{Forward tracker}

The forward tracker, containing similar straw tubes to the STT, assembled in three planar tracking stations, will be installed in the forward spectrometer to cover small polar angles up to $\pm 10^{\circ}$ horizontally and $\pm 5^{\circ}$ vertically. The momentum acceptance is larger than $3 \%$ of the beam momentum. This goal is achieved by adjusting the dipole field according to the beam momentum. For the position resolution, $0.1 \mathrm{~mm}$ per layer can be achieved, whereas the momentum resolution will be better than $1 \%$.

\section{Particle Identification}

The envisaged physical programs of PANDA require excellent PID for all decay channels. Since PANDA does not comprise hadronic calorimeters, the PID of hadrons will be performed with four different detector methods: 2 Cherenkov detectors, a Time of Flight (ToF) system, the specific energy loss from the STT and MVD, and a muon detection system.

ISSN 2071-0194. Ukr. J. Phys. 2019. Vol. 64, No. 7 


\subsection{Barrel DIRC}

One of the Cherenkov detectors based on the Detection of Internally Reflected Cherenkov Light (DIRC), is the Barrel DIRC [9] which will be mounted in a cylindrical shape around the STT. It is designed to separate $\pi^{ \pm}$and $K^{ \pm}$with a separation power of more than 3 s.d. in the polar angle range of $22^{\circ}$ to $140^{\circ}$ and the momentum range of 1.5 to $3.5 \mathrm{GeV} / \mathrm{c}$.

The radius of the detector is $476 \mathrm{~mm}$. It will consist of 16 fused silica bars and 128 Multichannel Plate Photomultiplier Tubes (MCP-PMTs), which adds up to around 10,000 channels to be read out with the DiRICH readout system. The MCP-PMT signal shape results in a time resolution of $100 \mathrm{ps}$.

\subsection{Disc DIRC}

Another Cherenkov detector called Disc DIRC [6] will be placed at the forward endcap of the PANDA target spectrometer, around $2 \mathrm{~m}$ away from the interaction point in the downstream direction. It will cover small polar angles between $5^{\circ}$ and $22^{\circ}$ and particle momenta of $\pi^{ \pm}$and $K^{ \pm}$between 0.5 and $4.0 \mathrm{GeV} / \mathrm{c}$. As for the Barrel DIRC, the separation power will be larger than 3 s.d. The Disc and Barrel DIRC together will cover almost the full kaon phase space in the target region.

The Disc DIRC consists of four independent quadrants made of synthetic fused silica. The detector radius is approx. 1,200 mm. Currently, 96 MCP-PMTs are foreseen for the photon detection, which requires a readout of 30,000 channels with TOFPET ASICs from the company PETsys. The time resolution is similar to the one of the Barrel DIRC. For the reconstruction of the Cherenkov angle, the tracking information has to be taken additionally into account.

\subsection{Barrel ToF}

The Barrel ToF [1], also called SciTil detector, is required for the PID of low momentum particles below $1 \mathrm{GeV}$. A very good time resolution of better $100 \mathrm{ps}$ is required for that purpose and can be achieved with a high photon yield. In total, 5,760 scintillator tiles with sizes around $90 \mathrm{~mm} \times 30 \mathrm{~mm} \times 5 \mathrm{~mm}$ have to be installed in the target spectrometer around the Barrel DIRC.

The scintillator material has not been finally chosen, but it will be either EJ-228 or EJ-232 from Eljen Technology. The photon signals will be detected with
Silicon Photomultipliers (SiPMs) that can be read out in combination with PETsys TOFPET ASICs.

\subsection{Forward ToF}

In the forward spectrometer, PID is essential. Hence, a forward ToF system was developed. It does not require a start counter, but uses relative timing to the Barrel ToF. The baseline design of the Forward ToF is a wall of scintillator slabs. In the center of the detector, 20 slabs with the dimensions $1400 \mathrm{~mm} \times$ $\times 5 \mathrm{~mm} \times 2.5 \mathrm{~mm}$ will be used. On both sides, the width of each slap changes from $5 \mathrm{~cm}$ to $10 \mathrm{~cm}$. BC409, made by Saint-Gobain Crystals, will be used as a scintillation material. The photon detection will be done with 1-inch PMTs R4998 from Hamamatsu that are going to be mounted on both ends. The detector is going to be installed $7.5 \mathrm{~m}$ away from the interaction point.

\subsection{Forward RICH}

The PID detector with the largest distance to the interaction point is the forward Ring Imaging Cherenkov (RICH) that is placed behind the Forward ToF for phase 2. It contains two layers of aerogel with small refractive indices of $n_{1}=1.05$ and $n_{2}=1.047$ in order to obtain a better focusing of the Cherenkov ring. The setup is very simple, because it contains only one flat mirror and a Multi Anode PMT (MaPMT) array with a pixel size of $6 \mathrm{~mm}$ to determine the position of the photon hits. A separation of $\pi^{ \pm} / K^{ \pm}$and $\mu^{ \pm} / K^{ \pm}$up to particle momenta of $10 \mathrm{GeV} / \mathrm{c}$ is realistic. The used MaPMTs are approx. 10 times less expensive than MCP-PMTs, but still have a long lifetime and a sufficient radiation hardness.

\section{Energy Measurement}

PANDA contains Electromagnetic Colorimeters (EMCs) in the target and a forward spectrometer with the goal to achieve a good energy and spatial resolution for photons from a few $\mathrm{MeV}$ up to $15 \mathrm{GeV}$ in order to reconstruct almost all multiphoton and lepton-pair channels. Because of the hadronic interactions in PANDA, a high photon yield and the background suppression are required. For that purpose, the energy threshold of all calorimeters has to be set to a value around $10 \mathrm{MeV}$. The rate of a single crystal is given by $10^{6} \mathrm{~s}^{-1}$. 


\subsection{Target Calorimeter}

The EMC in the target spectrometer [8] contains one barrel part, which will be installed around the Barrel ToF, and two flat parts at the forward and backward endcaps. It uses the $2^{\text {nd }}$ generation of $\mathrm{PbWO}_{4}$ crystals with improved light yield and better radiation hardness. In total, 15,744 crystals have to be installed in all parts. In order to increase the photon yield by a factor of four, the crystals have to be cooled to a temperature of $(-25 \pm 0.1){ }^{\circ} \mathrm{C}$. The used material has the advantage of a small radiation length around $0.9 \mathrm{~cm}$ and and a Molière radius of $2.1 \mathrm{~cm}$. The typical size of each crystal is $2.5 \mathrm{~mm} \times 2.5 \mathrm{~mm}$ with a fixed length of $20 \mathrm{~cm}$. For particles with energies above $100 \mathrm{MeV}$, a time resolution of better than $1 \mathrm{~ns}$ and a spatial resolution of less than $1.5 \mathrm{~mm}$ are feasible. The energy resolution is given by

$\frac{\sigma(E)}{E}=1 \% \oplus \frac{2 \%}{\sqrt{E[\mathrm{GeV}]}}$.

Currently, $75 \%$ of all required crystals have been produced.

\subsection{Forward calorimeter}

The EMC in the forward spectrometer [10] is a shashlyk-type sampling calorimeter consisting of interleaved scintillators and lead absorbers. The photon readout is done with PMTs and FADCs that are used for the signal digitization. The active area of the calorimeter is given as $297 \times 154 \mathrm{~cm}^{2}$. The total energy resolution can be calculated to

$\frac{\sigma(E)}{E} \leq 1.3 \% \oplus \frac{2.8 \%}{\sqrt{E[\mathrm{GeV}]}} \oplus \frac{3.5 \%}{E[\mathrm{GeV}]}$.

\section{Muon Detector System}

In the iron yoke of the target spectrometer and in the forward spectrometer, small Muon Drift Tubes (MDTs) with a wire and cathode strip readout will be used to detect created muons. Due to the low muon momenta, a large pionic background is expected. This effect can be minimized by using a multilayer range system. In PANDA, $12+2$ layers are installed in the barrel and $5+2$ layers in the endcap part. Between the target and forward spectrometer, muon filters will be installed for the background reduction. Behind the forward EMC, $16+2$ layers of muon chambers will be installed additionally. In total, the setup will contain 3,751 MDTs in all parts.

\section{Luminosity Detector}

The luminosity detector of PANDA will be placed around $11 \mathrm{~m}$ away from the target in the forward direction behind the forward spectrometer. It is going to measure the elastic scattering of antiproton-proton interactions. The main component is a silcon pixel detector that is mounted on five Chemical Vapour Deposition (CVD) diamond wafers with a thickness of $200 \mu \mathrm{m}$. Each wafer contains 10 High Voltage Monolithic Active Pixel Sensors (HV MAPS) with a pixel size of $80 \mu \mathrm{m} \times 80 \mu \mathrm{m}$. The active pixel sensor is based on the CMOS technology which allows the digital processing directly on a chip. The detector is able to attain a very high efficiency of approx. 99.5\%.

\section{Hypernuclear Setup}

The hypernuclear setup is an alternative setup for physical studies in phase 2. It contains two targets: one passive primary target, made of a diamond wire on piezo motored wire holders, to produce $\Xi$ baryons and one secondary active target for capturing them together with all track products in silicon microstrips and absorbers. High-purity germanium detectors at the rear will be used for gamma spectroscopy of the related decay products.

\section{Data Acquisition}

One of the outstanding features of PANDA is the triggerless data acquisition [12]. Because of the absence of a hardware trigger, the data from the Front End Electronics (FEE) have to be reduced by a factor of more than 1,000. This reduction will be achieved by a daisy chain of different event building and online reconstruction levels. First, the data from the FEE will be transmitted via data concentrators to a burst building network. From there, the remaining hits will be processed further in special compute nodes to perform the $1^{\text {st }}$ and $2^{\text {nd }}$ level selections, before the reduced data will be written to the PANDA storage. The time synchronization will be done with a dedicated PANDA development called SODAnet.

\section{Simulation Framework}

For the simulation and analysis, a dedicated framework called PandaRoot [11], that is based on ROOT, was developed. This framework includes the geometries of all PANDA subdetectors together with the important simulation parameters and passive volumes. Different particle generators can be used in or-

ISSN 2071-0194. Ukr. J. Phys. 2019. Vol. 64, No. 7 


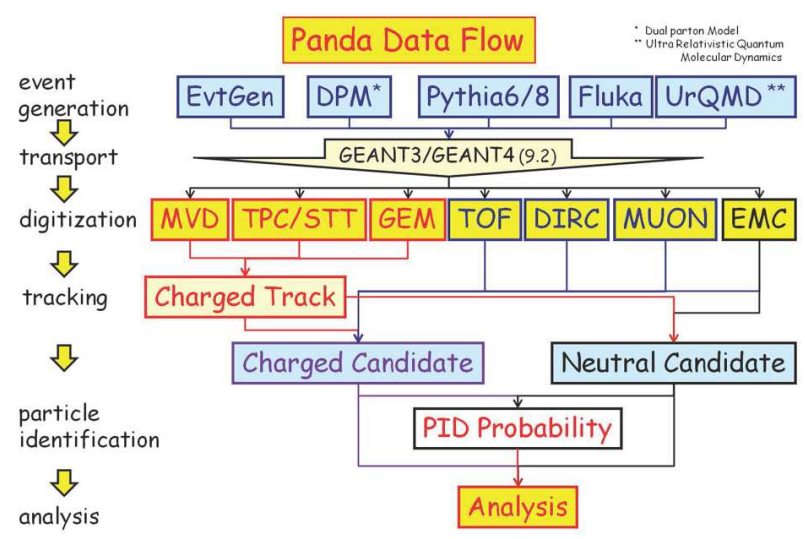

Fig. 3. The data flow in PandaRoot

der to simulate from dedicated probe tracks to specific physics channels. For the particle propagation through matter, it is possible to switch between the toolkits Geant3 and Geant4. The PandaRoot framework will additionally be used to reconstruct and analyze the acquired data of the final PANDA detector, as shown in Figure 3.

1. M. Böhm et al. The PANDA barrel-tof detector. Nuclear Instruments and Methods in Physics Research Section A: Accelerators, Spectrometers, Detectors and Associated Equipment 912, 323 (2018).

2. M. Destefanis. The PANDA experiment at FAIR. In: The Proceedings of the 7th Joint International Hadron Structure'13 Conference. Nucl. Phys. B - Proceedings Supplements 245, 199 (2013).

3. W. Erni et al. Technical Design Report for the PANDA Solenoid and Dipole Spectrometer Magnets. arXiv:0907.0169 [physics.ins-det] (2009).

4. W. Erni et al. Technical design report for the: PANDA micro vertex detector. arXiv:1207.6581 [physics.ins-det] (2012).
5. W. Erni et al. Technical design report for the PANDA (AntiProton Annihilations at Darmstadt) straw tube tracker. Eur. Phys. J. A 49, 25 (2013).

6. K. Föhl et al. The PANDA Endcap Disc DIRC. JINST 13 (02), C02002 (2018).

7. M.F.M. Lutz et al. Physics performance report for PANDA: Strong interaction studies with antiprotons. arXiv:0903.3905 [hep-ex] (2009).

8. M. Moritzet al. The electromagnetic calorimeter for the PANDA target spectrometer. J. Phys.: Conf. Series 1162, 012025 (2019).

9. B. Singh et al. Technical design report for the PANDA barrel Dirc detector. arXiv:1710.00684 [physics.ins-det] (2017).

10. B. Singh et al. Technical design report for the PANDA forward spectrometer calorimeter. arXiv:1704.02713v1 [physics.ins-det] (2017).

11. S. Spataro, the PANDA Collaboration. The pandaroot framework for simulation, reconstruction and analysis. J. Phys.: Conf. Series 331 (3), 032031 (2011).

12. H. Xu, Z.-A. Liu, Q.Wang, W. Kuehn, S. Lang, M. Liu. Introduction to panda data acquisition system. In: Proceedings of the 2nd International Conference on Technology and Instrumentation in Particle Physics (TIPP 2011). Phys. Proc. 37, 1855 (2012).

Received 08.07.19

M. Шмiдm

\section{ДЕТЕКТОР РАNDА НА ПРИСКОРЮВАЧІ FAIR}

$\mathrm{P}$ е $з$ ю м е

PANDA - експеримент з фіксованою мішенню, в якому передбачається розглянути широкий спектр відкритих питань адронної фізики шляхом дослідження взаємодії між антипротонами з великими імпульсами та стаціонарною протонною мішенню. Детектор PANDA наразі знаходиться на стадії будівництва і буде розміщений у HESR, що є частиною майбутнього комплексу прискорювача FAIR на платформі Центру Гельмгольца для дослідження важких іонів GSI у Дармштадті. Головні характеристики детектора: треки високої точності в сильному магнітному полі, чудова ідентифікація частинок, а також калориметри високої роздільної здатності. 